\title{
Avaliação qualitativa de dentes artificiais de resina acrílica após escovação simulada
}

\author{
Qualitative evaluation of artificial acrylic resin teeth after simulated brushing
}

\author{
Cássia Souza Fernandes ${ }^{1 *}$, Ravanna Silva Muniz² ${ }^{2}$ Marcelle Alvarez Rossi ${ }^{3}$, Danilo Barral de Araujo ${ }^{4}$ \\ ${ }^{1}$ Mestranda do Programa de Pós-graduação Processos Integrativos dos Órgãos e Sistemas, Instituto de Ciências da \\ Saúde, Universidade Federal da Bahia-UFBA; ${ }^{2}$ Acadêmica do Curso de Graduação em Odontologia, Faculdade de \\ Odontologia - UFBA; ${ }^{3}$ Professora Adjunto, Instituto de Ciências da Saúde - UFBA; ${ }^{4}$ Professor Associado, Instituto de \\ Ciências da Saúde, Programa de Pós-graduação em Processos Interativos dos Órgãos e Sistemas - UFBA
}

\begin{abstract}
Resumo
Introdução: os dentes artificiais de resina acrílica são amplamente utilizados na confecção de próteses, por apresentarem propriedades físicas, mecânicas e biológicas, além de características de fácil manuseio. No entanto, devido sua característica de baixa resistência à abrasão, a escovação pode levar à perda de massa dos dentes artificiais e aumento da rugosidade. Objetivo: avaliar de forma qualitativa a rugosidade superficial e polimento de dentes artificiais das marcas Hereaus Premium; Trilux e Vita, após período de 1 ano de escovação, com fotografias em Lupa Estereoscópica. Metodologia: para a realização deste estudo, serão utilizados 10 dentes artificiais de cada marca comercial (Trilux, Vita e Hereaus Premium). Os dentes serão fixados com resina ortoftálica em tubo de PVC de $15 \mathrm{~mm}$ de espessura. Para o teste de abrasão, será usada uma máquina de escovação simulada com escovas de dentes de cerdas macias e solução de pasta de dente. As imagens serão obtidas com câmera digital acoplada a lupa estereoscópica. A rugosidade superficial e o polimento serão analisados antes da escovação simulada e após 12 meses de escovação através das imagens. Resultados: após o teste de abrasão foi observado a presença de um desgaste superficial e/ou ranhuras na superfície dos dentes artificiais em todos os grupos. Porém não houve um padrão similar entre os corpos de prova de um mesmo grupo. Conclusão: Pode-se concluir que a escovação provocou o aumento da rugosidade superficial e alteração no polimento em todos os dentes artificiais utilizados. Palavras-chave: Dente artificial. Abrasão Dentária. Resinas acrílicas.
\end{abstract}

\begin{abstract}
Introduction: acrylic resin artificial teeth are widely used in the manufacture of dentures, as they have physical, mechanical and biological properties, in addition to easy handling characteristics. However, due to its characteristic of low abrasion resistance, brushing can lead to loss of mass of artificial teeth and increase in roughness. Objective: qualitatively evaluate the surface roughness and polishing of artificial teeth of Hereaus Premium brands; Trilux and Vita, after a 1-year brushing period, with photographs using a Stereoscopic Magnifying Glass. Methodology: for this study, 10 artificial teeth of each commercial brand will be used (Trilux, Vita and Hereaus Premium). Teeth will be fixed with orthophthalic resin in a $15 \mathrm{~mm}$ thick PVC tube. For the abrasion test, a simulated brushing machine with soft bristle toothbrushes and toothpaste solution will be used. Images will be obtained with a digital camera coupled to a stereoscopic magnifying glass. Surface roughness and polishing will be analyzed before simulated brushing and after 12 months of brushing through the images. Results: after the abrasion test, the presence of surface wear and/ or grooves on the surface of the artificial teeth was observed in all groups. However, there was no similar pattern between the specimens of the same group. Conclusion: it can be concluded that brushing caused an increase in surface roughness and change in polishing in all artificial teeth used. .

Keywords: Artificial Tooth. Tooth Abrasion. Acrylic Resins.
\end{abstract}

\section{INTRODUÇÃO}

Os dentes artificiais de resina acrílica são muito utilizados em próteses por apresentarem vantagens, como melhor absorção de forças mastigatórias, aparência mais natural, melhor união com a resina da base da prótese, maior facilidade de ajustes estéticos e funcionais, além de fácil polimento. Porém, apresentam uma baixa resistência

Correspondente/Corresponding: *Cássia Souza Fernandes - End: Alameda das Nações, 53, Nova Brasília, Salvador, BA. - Tel: (71) 992449889 - E-mail: cassiasouza15@hotmail.com à abrasão e baixa estabilidade de cor, o que pode levar ao desequilíbrio oclusal e problemas estéticos (BRIGAGÃO; CAMARGO; NEISSER, 2005; DUARTE et al., 2006; OLIVEIRA et al., 2013; TABATA et al., 2006).

No estudo das propriedades físicas dos dentes de resina acrílica, é importante avaliar a abrasão e a rugosidade superficial causada pela escovação. A abrasão pode levar à perda de massa dos dentes artificiais, o que compromete a adaptação da prótese, e da estética, bem como pode causar um aumento da rugosidade. A rugosidade superficial aumentada predispõe a um maior acúmulo 
de biofilme, já a menor rugosidade reduz a retenção de microrganismos, previne infecções locais e a deterioração da prótese (PISANI et al., 2010; SORGINI, 2011).

A escovação com cremes dentais pode alterar a superfície do material por meio de abrasão e atrição, com um aumento da rugosidade da superfície. Existem diversos fatores associados à escovação que podem afetar o grau de abrasão dos dentes de resina acrílica, como a dureza da resina acrílica, o tipo do agente abrasivo, a forma e o tamanho das partículas abrasivas, o grau de diluição do dentifrício, o tipo de escova de dentes usada e a força aplicada. (FREITAS; PARANHOS, 2006; PISANI et al., 2010).

$O$ ideal é que a superfície das próteses apresente-se adequadamente lisa e polida, para sua maior longevidade. Nesse contexto, o objetivo deste estudo foi avaliar de forma qualitativa a rugosidade superficial e o polimento de dentes artificiais das marcas Hereaus Premium, Trilux e Vita, após período deum ano de escovação, com fotografias em lupa estereoscópica.

\section{METODOLOGIA}

\section{Seleção e Preparo dos Corpos de Prova}

Para a realização deste estudo, utilizaram-se três marcas comerciais de dentes artificiais de resina acrílica, quais sejam, Trilux (Dental Vipi, Pirassununga, SP, Brasil), Vita (VITA Zahnfabrik, Bad Sãckingen, Germany) e Hereaus Premium (Heraeus Kulzer South America Ltda, São Paulo, $\mathrm{SP}, \mathrm{Brasil})$. De cada marca comercial, foram utilizados 10 dentes artificiais, incisivos centrais superiores.

Um tubo de PVC da marca Tigre foi seccionado em pedaços de $15 \mathrm{~mm}$ e aplainados com lixa 320 (NDR), tendo sido usados como moldes para confecção dos corpos de prova. Os canos foram isolados com vaselina líquida (ADV Farma), com auxílio de um pincel (Tigre - $n$ - 267), para evitar que a resina aderisse às paredes internas, tendo um dos lados sido preenchido com cera utilidade na cor vermelha (Technew), para que a resina não vazasse durante sua presa, além, é claro, da preservação das faces vestibulares das unidades dentais, alvo deste estudo.

Figura 1-Dentes inseridos na resina ortoftálica no tubo de PVC, aguardando o tempo de presa.

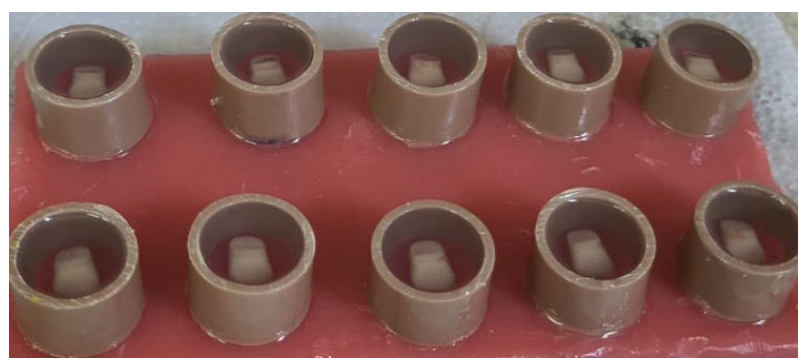

Fonte: Foto de arquivo dos autores.

Um copo plástico transparente de $200 \mathrm{ml}$ foi utilizado para manipulação da resina. Neste recipiente, colocaram-se 110 gramas da resina ortoftálica transparente
(Centerglass), com 22 gotas do catalisador (seguindo orientações do fabricante) e com uma espátula de madeira manipulou-se a mistura obtendo o ponto ideal da resina para realizar as inserções dos dentes. Um período de 24 horas foi necessário para a presa completa da resina, seguindo instruções do fabricante (Figura 1).

Após o preparo individual das unidades, realizaram-se as imagens iniciais com câmera digital (Moticam ${ }^{\circledR}, 3.0 \mathrm{MP}$ ) acoplada à lupa estereoscópica (Motic ${ }^{\circledR}$ ) no aumento de 0,7 e $1,0 \times$ (Figura 2).

Figura 2 - Realização das imagens do corpo de prova com a câmera digital acoplada à lupa estereoscópica.

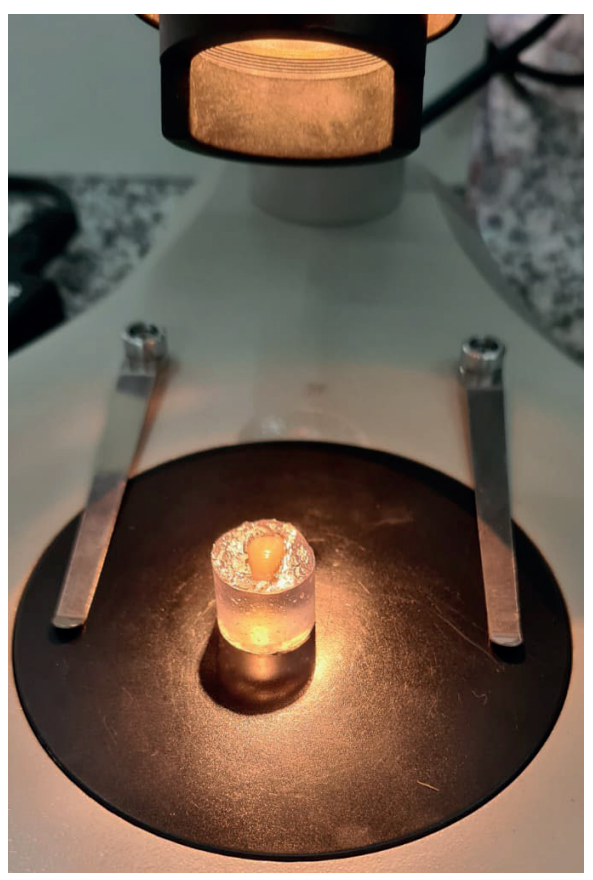

Fonte: Foto de arquivo dos autores.

\section{Teste de abrasão}

A máquina utilizada para escovação simulada (ElQuip $^{\circledR}$, São Paulo, SP, Brasil) apresentou velocidade de 4,5 ciclos/seg., em movimentos de vai-e-vem. Utilizaram-se "cabeças" das escovas dentárias de cerdas macias (Essencial Clean/Colgate-Palmolive Company ${ }^{\circledR}$ São Paulo, Brasil) para escovação dos corpos de prova. As soluções de dentifrícios (Colgate Total 12/ Colgate-Palmolive Company ${ }^{\circledR}$ São Paulo, Brasil) foram preparadas na proporção de 1:2, segundo metodologia de Tao et al. (2017) e submetidas à verificação de $\mathrm{pH}$ (pHmetro Modelo 2000 Quimis $^{\circledR}$ Aparelhos Científicos Ltda., São Paulo, Diadema, Brasil). A máquina realizou 50 mil ciclos de escovação, para simular um ano de uso da prótese, levando em consideração a escovação três vezes ao dia (Figura 3). 
Figura 3 - Teste de abrasão realizado na máquina de escovação com 10 corpos de prova.

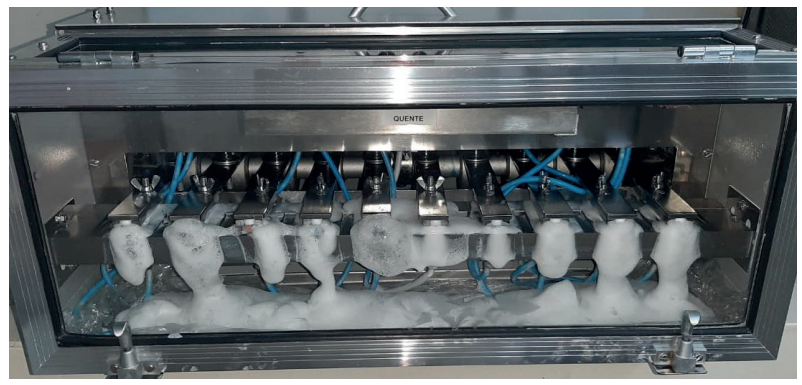

Fonte: Foto de arquivo dos autores.

Após o teste de escovação, os corpos-de-prova foram lavados em cuba ultrassônica por 10 minutos com água destilada (Figura 4), para realização das imagens finais com a câmera digital (Moticam $^{\circledR}, 3.0 \mathrm{MP}$ ), acoplada à lupa estereoscópica (Motic ${ }^{\circledR}$ ), no aumento de 0,7 e 1,0 X em todos os grupos.

Figura 4 - Lavagem dos corpos de prova em cuba ultrassônica, após a escovação.

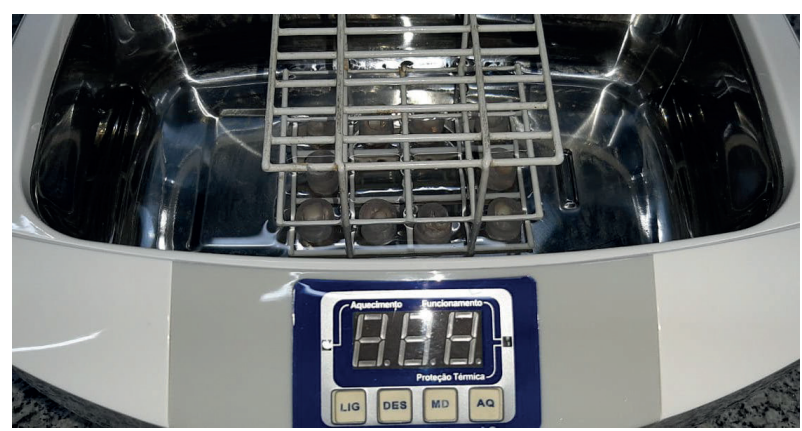

Fonte: Foto de arquivo dos autores.

As superfícies vestibulares dos dentes artificiais, área submetida ao teste de abrasão, foram analisadas e comparadas com a imagem inicial de cada corpo de prova, antes da escovação simulada.

\section{RESULTADOS}

Observaram-se características similares entre os grupos nas fotos iniciais, com um padrão similar de lisura e polimento (Figuras 5, 7 e 9). Após o teste de abrasão, notou-se a presença de um desgaste superficial e/ou ranhuras na superfície dos dentes artificiais (Figuras 6, 8 e 10).

Segundo revelam as imagens, há uma diferença entre os grupos: os dentes Vita e Premium mostraram-se com maior desgaste e rugosidade superficial nas imagens (Fi- guras 6 e 10). Contudo, não houve um padrão similar entre os corpos de prova do mesmo grupo, sendo que alguns se apresentaram com maior desgaste e já outros com poucas ranhuras vistas nas imagens (Figuras 10, 11 e 12). Os dentes Trilux tiveram um padrão similar nas imagens, com pouca diferença de desgaste e rugosidade percebida entre as imagens iniciais e finais (Figuras 7 e 8, 13 e 14).

Figura 5 - Dente Vita (corpo de prova 1), fotografia inicial, no aumento 1x. A fotografia mostra a face vestibular lisa, polida, com sulcos característicos da anatomia do incisivo central superior.

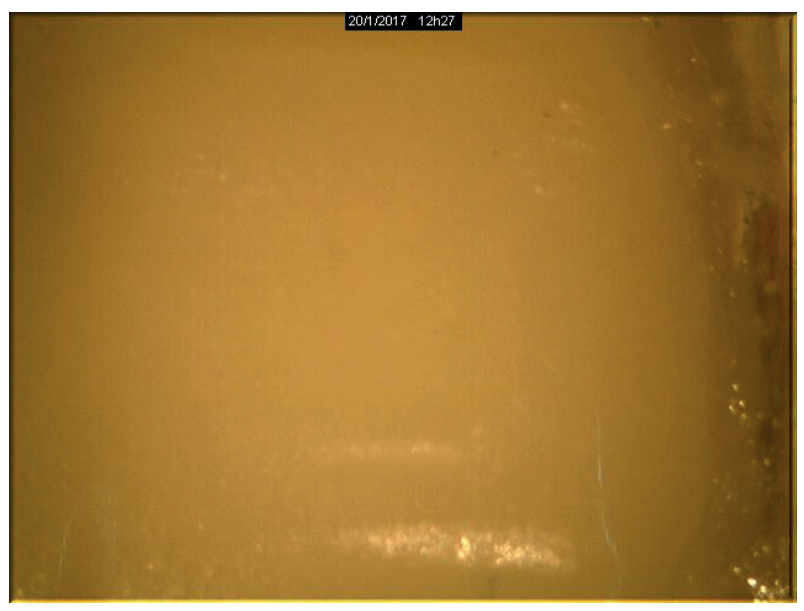

Fonte: Foto de arquivo dos autores.

Figura 6 - Dente Vita (corpo de prova 1), fotografia final, no aumento 1x. Presença de ranhuras, estrias e diminuição do brilho na superfície da face vestibular do dente.

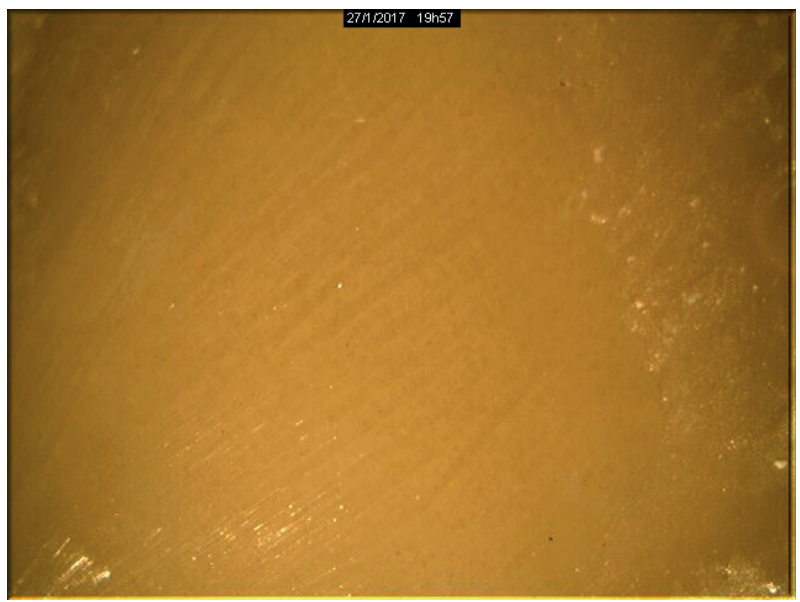

Fonte: Foto de arquivo dos autores. 
Figura 7 - Dente Trilux (corpo de prova 1), fotografia inicial, no aumento $1 x$. A face vestibular mostra-se lisa e polida, com padrão regular.

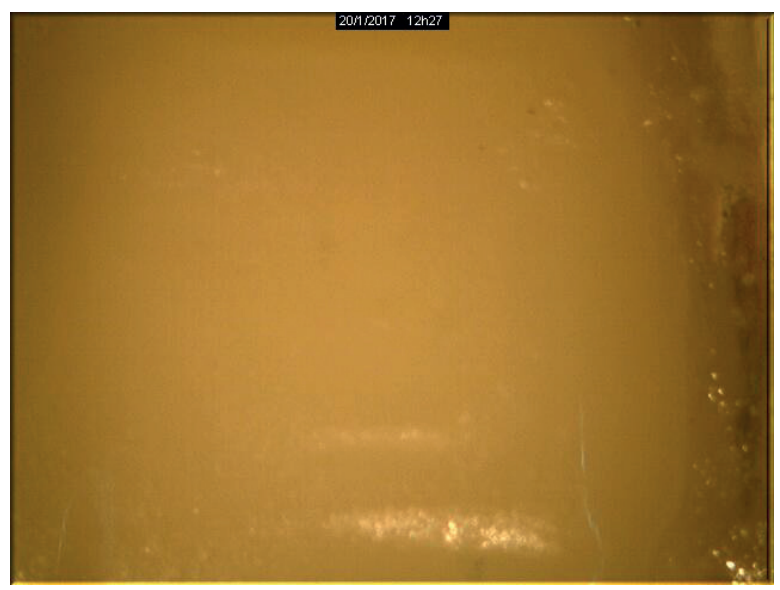

Fonte: Foto de arquivo dos autores.

Figura 8 - Dente Trilux (corpo de prova 1), fotografia final, no aumento 1x. A imagem mostra a face vestibular com presença de ranhuras, com irregularidades na superfície.

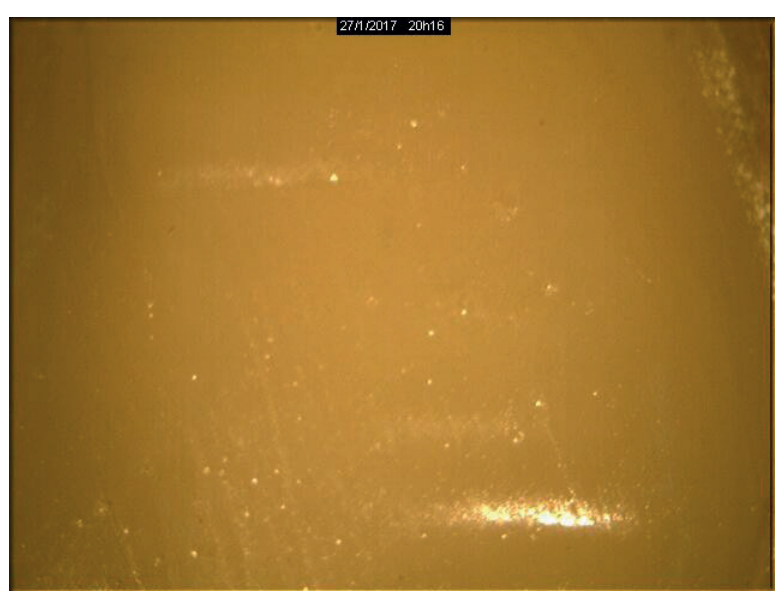

Fonte: Foto de arquivo dos autores.

Figura 9 - Dente Premium (corpo de prova 1), fotografia inicial, no aumento 1x. A face vestibular mostra-se lisa e polida, com padrão regular.

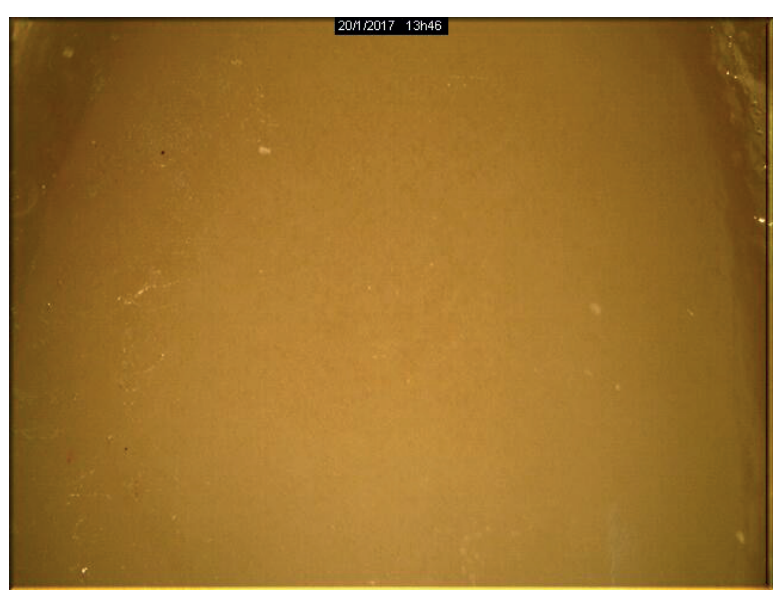

Fonte: Foto de arquivo dos autores.
Figura 10 - Dente Premium (corpo de prova 1), fotografia final, no aumento $1 x$. A imagem mostra a presença de irregularidades, com estrias e ranhuras na superficie.

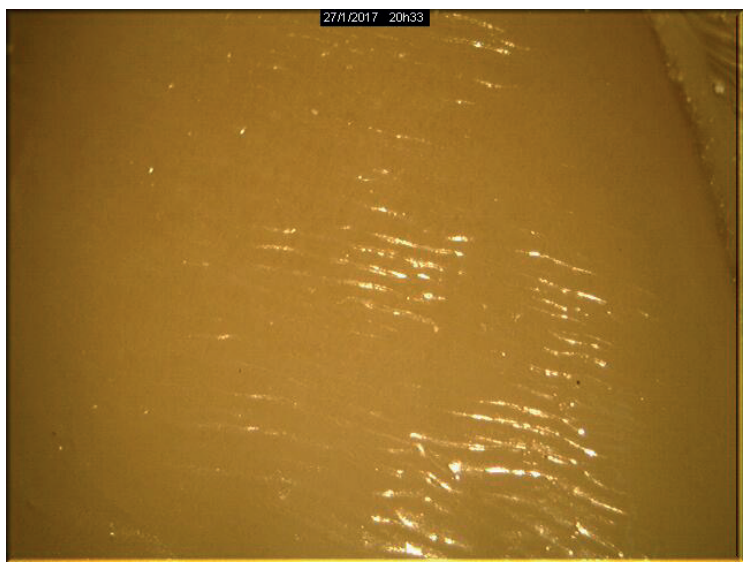

Fonte: Foto de arquivo dos autores.

Figura 11 - Dente Premium (corpo de prova 2), fotografia final, no aumento $1 x$. A imagem mostra a presença de ranhuras $e$ estrias na superficie.

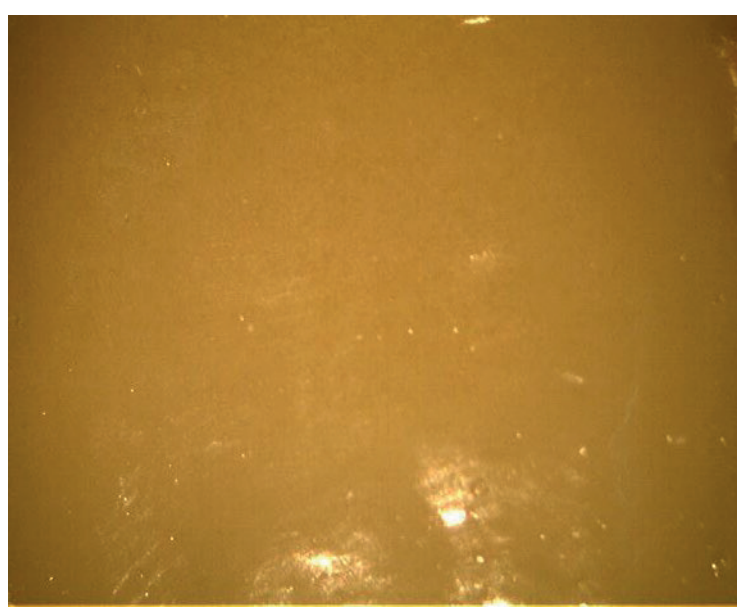

Fonte: Foto de arquivo dos autores.

Figura 12 - Dente Premium (corpo de prova 8), fotografia final, no aumento $1 x$. A imagem mostra a presença de ranhuras na superficie.

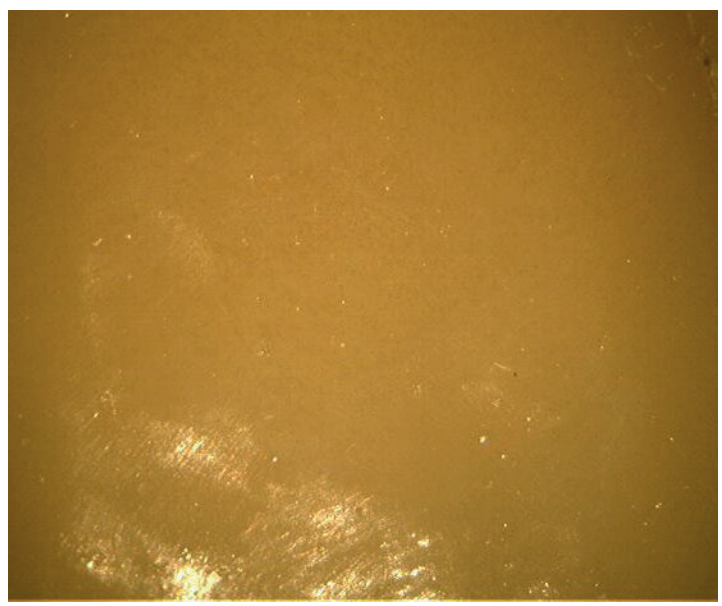

Fonte: Foto de arquivo dos autores. 
Figura 13 -Dente Trilux (corpo de prova 7), fotografia inicial, no aumento 1x. A imagem mostra a face vestibular com polimento e lisa.

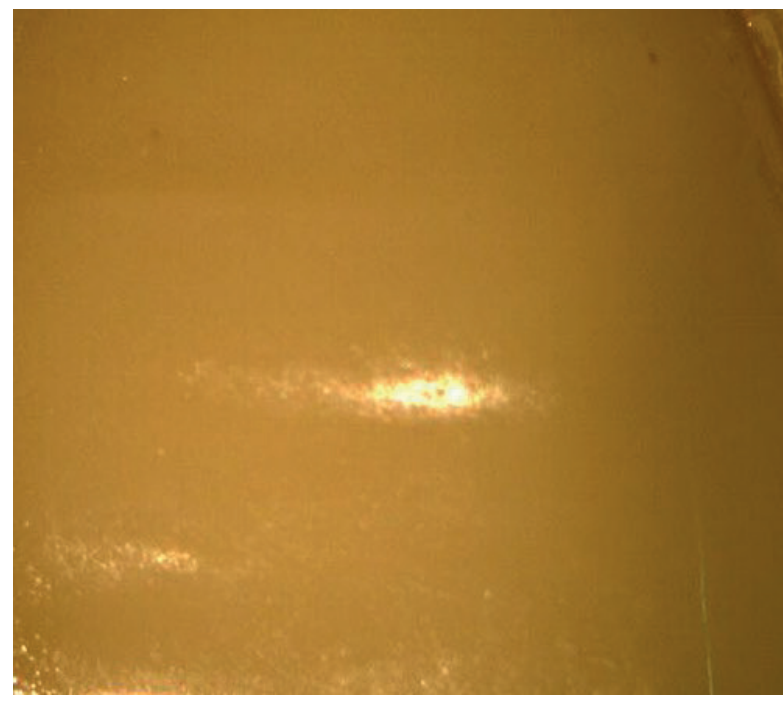

Fonte: Foto de arquivo dos autores.

Figura 14 - Dente Trilux (corpo de prova 7), fotografia final, no aumento $1 x$. A imagem mostra a face vestibular com pouca diferença da imagem inicial.

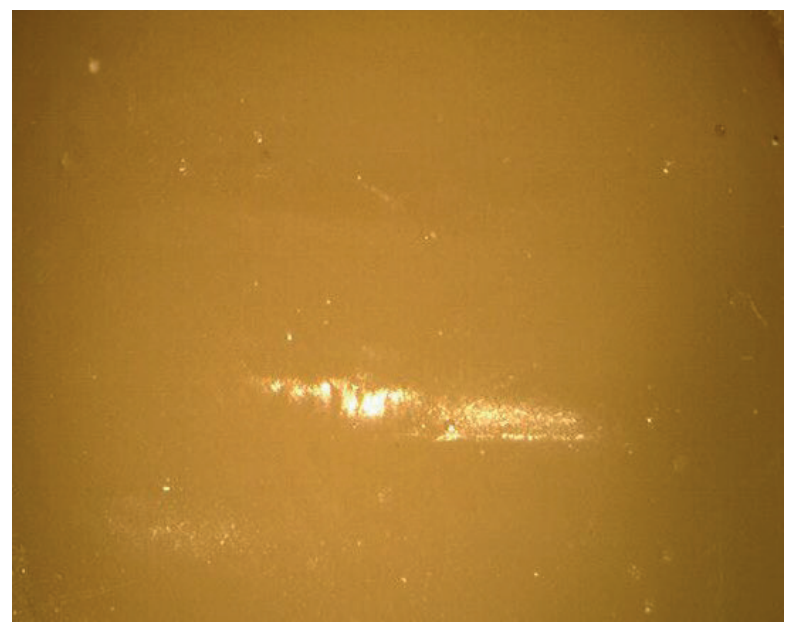

Fonte: Foto de arquivo dos autores.

\section{DISCUSSÃO}

Estudos prévios mostram que os métodos de higiene, como a escovação, podem interferir na integridade da superfície dos dentes artificiais de resina acrílica (FREITAS; PARANHOS, 2006; GRANDO et al., 2015; OLIVEIRA et al., 2013). A escovação pode provocar abrasão e um aumento da rugosidade superficial e esses fatores podem levar à perda de massa dos dentes artificiais, o que compromete a adaptação da prótese e a estética (PISANI et al., 2010; SORGINI, 2011). Os resultados encontrados no presente estudo estão em concordância com a literatura, visto que a escovação simulada equivalente a um ano foi fator contribuinte para a abrasão, aumento da rugosidade superficial percebida nas imagens (Figuras 5, 7, 9 e 11) e, consequentemente, prejuízo para a estética dos dentes.

A presença de irregularidades na superfície, que caracteriza o aumento da rugosidade superficial, foi encontrada neste estudo após a escovação, corroborando outros estudos (BRIGAGÃO; CAMARGO; NEISSER, 2005; FREITAS; PARANHOS, 2006; GRANDO et al., 2015; OLIVEIRA et al., 2013; PISANI et al., 2010; SORGINI, 2011;), e mostrando, assim, que a integridade da resina acrílica pode ser danificada pela escovação, com aumento da rugosidade e perda de polimento.

Há uma relação direta entre a rugosidade e o brilho da superfície (SAKAGUCH et al., 1986), pois, quanto maior a homogeneidade da superfície, maior a capacidade da estrutura emitir brilho. A interação entre luz e objeto está relacionada com características físicas e óticas, o que pode interferir na percepção da cor, assim como a presença de diferença de textura e curvatura na superfície (ARAKAKI; VIEIRA, 2010). Após o teste de abrasão realizado nos dentes artificiais no presente estudo, percebeu-se aumento da rugosidade (Figuras 5, 7 e 9), com presença de irregularidades na superfície e alteração no polimento, diferente do estado inicial dos dentes, antes da escovação, o que revela que a presença de irregularidades na superfície do dente altera a sua estética.

No estudo de Arakaki e Vieira (2010) com resinas compostas, houve diferença de percepção de cor entre corpos de prova com superfície lisa e superfície rugosa, sendo assim identificada a relação entre aumento da rugosidade superficial e a variação da cor dos materiais restauradores. Apesar da diferença de metodologia e material, a rugosidade superficial presente nos corpos de prova das resinas compostas do estudo abordado mostrou-se fator para mudança na percepção do brilho e da cor do material. Esta condição é importante, pois a estética das próteses pode ser afetada com a alteração de brilho e lisura, após o aumento da rugosidade superficial, conforme ocorreu em todos os grupos.

As escovas e os dentifrícios têm o potencial de trazer danos à superfície dos dentes artificiais, independentemente da qualidade do polimento inicial (BARRETO et al., 2019). Esta condição foi encontrada no presente estudo, visto que em todos os grupos de dentes, após o teste de abrasão, houve aumento da rugosidade e alteração no polimento percebido nas imagens (Figuras 5, 7 e 9).

A escovação pode ser prejudicial aos dentes de resina acrílica, causando desgaste e aumento da rugosidade de superfície pela ação das cerdas das escovas e também pela duração, frequência e força aplicada na escovação (SORGINI et al., 2015). Apesar da utilização de escovas macias neste trabalho, ainda assim houve a abrasão, que pode ser devido às características próprias da resina acrílica, por dispor de baixa resistência à abrasão, bem como pode estar associada ao uso de dentifrício, que contém agentes abrasivos, como a sílica, presente na composição do Colgate Total 12 (RIOS et al., 2014). Porém, a escova- 
ção mecânica é um método de higienização diária que permite a diminuição ou eliminação da placa bacteriana, essencial para a saúde bucal, sendo necessária a utilização de produtos eficazes e não prejudiciais aos materiais do dispositivo protético, para evitar o aumento da porosidade e da rugosidade da resina acrílica das próteses, que leva à alteração de cor e resistência (PARANHOS et al., 2007).

Visto que os dentes de resina acrílica sofreram abrasão causada pela escovação e que este método de higiene é fundamental para a saúde bucal, são necessários procedimentos de acabamento e polimento de forma periódica, visando à manutenção da estética e da obtenção de próteses lisas e polidas (TUPINAMBÁ et al., 2018).

\section{CONCLUSÃO}

O estudo com dentes artificiais apresenta algumas dificuldades ao ser comparado com resultados de outros trabalhos, por ter diferenças entre as marcas comerciais, a carga da escovação, o dentifrício e o tipo de escova utilizada.

Neste estudo foi possível concluir que:

- Houve um aumento da rugosidade superficial após um ano de escovação simulada;

- As escovas, juntamente com o dentifrício, são agentes de abrasão e provocaram o aumento da rugosidade superficial e a alteração no polimento superficial dos dentes;

- O aumento da rugosidade superficial diminuiu a lisura da superfície do dente, porém, não houve uma alteração significativa no brilho desta estrutura.

São necessários mais estudos com uma análise qualitativa sobre o efeito da escovação no polimento e na alteração do brilho, que não se concentrem apenas na avaliação da rugosidade da superfície ou perda de massa.

AGRADECIMENTOS

Os autores agradecem a equipe do Laboratório de Bioquímica Oral (LBO) do Instituto de Ciências da Saúde da Universidade Federal da Bahia, pelo apoio na realização do estudo laboratorial.

\section{REFERÊNCIAS}

ARAKAKI, Y.; VIEIRA, G. F. Influência da rugosidade superficial de dentes artificiais de resina composta na percepção da cor entre profissionais da Odontologia. RPG Rev. Pós-Grad., São Paulo, v. 17, n. 4, p.197-203, 2010.

BARRETO J.O. et al. The effect of a continuous mechanical polishing protocol on surface roughness, biofilm adhesion, and color stability of acrylic resin artificial teeth. J Prosthodont., Fortaleza, v. 28, n. 1, p. 110-117, 2019.

BRIGAGÃO, V.C.; CAMARGO, F.P.; NEISSER, M.P. Avaliação in vitro da resistência ao desgaste de dentes artificiais. Cienc Odontol Bras., São José dos Campos, I. 8, n. 3, p. 55-63, jul./set. 2005.

DUARTE, A.R.C. et al. Avaliação de rugosidade superficial e resistência flexional de quatro resinas acrílicas utilizadas para próteses sobre implante, em função imediata. UFES Rev. Odontol., Vitória, v. 8, n. 1, p. 37-45, jan./abr. 2006.

FREITAS, K.M.; PARANHOS, H.F.O. Weight loss of five commercially available denture teeth after toothbrushing with three different dentifrices. J. Appl. Oral Sci., Bauru, v. 14, n. 4, p. 242-246, 2006.

GRANDO, M. et al. Artificial teeth: evaluation of wear resistance, microhardness and composition. Rev. Gaúcha Odontol., Campinas, v. 63, n. 3, p. 263-270, jul./set. 2015.

OLIVEIRA, A.S; RODRIGUES C.R.T.; BRUNO M.V.; CASTRO S.H.D. Análise da rugosidade e resistência à abrasão de três tipos de dentes artificiais em acrílico. Rev. UNINGÁ Rev., Maringá, v. 15, n. 1, p. 55-60, jul./set. 2013.

PARANHOS, H.F.O. et al. Effects of mechanical and chemical methods on denture biofilm accumulation. J. Oral Rehabil., Ribeirão Preto, v. 34, n. 8, p. 606-612, 2007.

PISANI, M. X. et al. Evaluation of the abrasiveness of dentifrices for complete dentures. J. Prosthod., Ribeirão Preto, vol. 19, n. 5, p. 369373, 2010.

RIOS, A. C. F. et al. Abrasivos, uma análise de dentifrícios comercializados em Salvador. Rev. Bahiana Odontol., Salvador, v. 5, n. 3, p. 141-152, 2014.

SAKAGUCHI, R. L. et al. The wear of a posterior composite in an artificial mouth: a clinical correlation. Dent. Mater., Minneapolis, v. 2, n. 6, p. $235-240,1986$.

SORGINI, D. B. Avaliação da perda de massa e alteração da rugosidade superficial causada pela associação dos métodos mecânico (escovação) e químico (imersão) de higiene de próteses totais. 2011. Dissertação (Mestrado em Reabilitação Oral) - Faculdade de Odontologia de Ribeirão Preto, Universidade de São Paulo, Ribeirão Preto, 2011.

SORGINI, D. B. et al. Adverse effects on PMMA caused by mechanical and combined methods of denture cleansing. Braz. Dent. J., Ribeirão Preto, v. 26, n. 3, p. 292-296, 2015.

TABATA, L.F. et al. In vitro abrasion resistance evaluation of different artificial resin teeth storaged in artificial saliva. Rev. Odontol. UNESP, Araraquara, v. 35, n. 4, p. 285-291, 2006.

TAO, D. et al. In vitro and clinical evaluation of optical tooth whitening toothpastes. J. Dent., [S.I.], v. 67, p. 25-28, 2017.

TUPINAMBÁ, Í. et al. Effect of different polishing methods on surface roughness of provisional prosthetic materials. J. Indian Prosthodontic Soc., Mumbai, v. 18, n. 2, p. 96-101, 2018.

Submetido em: 19/11/2021

Aceito em: 22/11/2021 